\title{
Síntomas de miastenia grave en un paciente con antecedente de timectomía por timoma invasor
}

\author{
Lilliana María Giraldo ${ }^{1,3}$, Camilo Duque $^{2}$, Carlos Santiago Uribe ${ }^{3}$, Olga Helena Hernández ${ }^{3}$ \\ 1 Especialización en Neurología Clínica, Facultad de Medicina, Universidad CES, Medellín, Colombia \\ 2 Unidad de Cuidados Intensivos, Clínica Cardiovid, Medellín, Colombia \\ ${ }^{3}$ Unidad de Cuidados Intensivos Neurológicos, Instituto Neurológico de Colombia, Medellín, Colombia
}

Introducción. La miastenia grave es una enfermedad autoinmunitaria mediada por anticuerpos. Entre 10 y $15 \%$ de quienes la padecen tienen timoma y su presencia se asocia con una mayor gravedad de los síntomas, crisis miasténicas y fracaso del tratamiento de primera línea. La timectomía se recomienda en pacientes jóvenes con miastenia grave generalizada y en todos los pacientes con timoma.

Caso clínico. Se presenta el caso de una mujer de 43 años que, en el 2005, presentó una primera crisis miasténica asociada con un timoma invasor que se trató con timectomía y radioterapia. Durante los siguientes tres años, presentó síntomas graves y dos crisis más, que obligaron a suministrarle respiración mecánica asistida e inmunoglobulina. Al cabo del tratamiento, no se evidenciaron signos de recurrencia en las tomografías de tórax con contraste. Entre el 2009 y el 2012, la gravedad de los síntomas fue menor. En el 2013, estos se exacerbaron y una resonancia magnética de tórax con contraste reveló una lesión en el mediastino anterior, ya observada en el 2011, sugestiva de tejido residual o fibrosis. Se inició el tratamiento usual con inmunoglobulina y se hizo una tomografía por emisión de positrones cuyos resultados no fueron concluyentes, por lo que se llevó a cabo una nueva resección y se constató que no había recurrencia del tumor.

Conclusiones. Los pacientes con miastenia grave y aquellos con timoma asociado, deben someterse a la timectomía como parte del tratamiento. Sin embargo, la exacerbación de los síntomas o su reaparición después del procedimiento no necesariamente implica una nueva alteración en el timo.

Palabras clave: miastenia grave, timoma, timectomía, tomografía de emisión de positrones, recurrencia. doi: http://dx.doi.org/10.7705/biomedica.v35i4.2756

Symptoms of myasthenia gravis in a patient with a history of thymectomy for invasive thymoma

Introduction: Myasthenia gravis is an antibody-mediated autoimmune disease. Approximately 10$15 \%$ of patients present with a thymoma, the presence of which is associated with greater severity of symptoms, myasthenic crisis, and irresponsiveness to front-line therapy. A thymectomy is recommended in young patients with generalized myasthenia gravis and in all patients presenting with thymoma.

Clinical case: The patient was a 43-year-old woman, who first showed symptoms of myasthenic crisis in 2005 and presented with invasive thymoma managed with thymectomy and radiotherapy. In the subsequent three years, the patient presented with severe symptoms and two myasthenic crises that required mechanical ventilation and immunoglobulin treatment. Contrast chest computed tomography examinations showed no recurrence. Between 2009 and 2012, the patient experienced decreased symptom severity. In 2013, the patient presented with an exacerbation of symptoms; a contrast chest magnetic resonance scan showed a lesion in the anterior mediastinum, previously observed in 2011, suggestive of residual tissue as opposed to fibrosis. Regular management was started with immunoglobulins; a positron emission tomography scan was inconclusive, requiring a new resection, which showed no evidence of tumor recurrence.

Conclusions: Patients with myasthenia gravis and those with myasthenia-related thymoma both share thymectomy as an element of treatment. However, following the procedure, exacerbation or reappearance of symptoms does not necessarily represent new alterations in the thymus.

Key words: Myasthenia gravis, thymoma, thymectomy, positron-emission tomography, recurrence. doi: http://dx.doi.org/10.7705/biomedica.v35i4.2756

\footnotetext{
Contribución de los autores:

Lilliana María Giraldo y Camilo Duque: búsqueda de la bibliografía

Lilliana María Giraldo: redacción del manuscrito

Camilo Duque, Carlos Santiago Uribe y Olga Helena Hernández: asesoría académica
} 
La miastenia grave es una enfermedad neurológica autoinmunitaria mediada principalmente por anticuerpos que actúan contra los componentes de la membrana muscular y la unión neuromuscular (1). En 80 a $85 \%$ de los casos los anticuerpos que actúan contra los receptores de la acetilcolina (AChR) son los responsables de alterar la transmisión neuromuscular (miastenia seropositiva), produciendo fatiga y debilidad muscular (2). En 20 a $40 \%$ de los pacientes con miastenia grave seronegativa, se han identificado recientemente nuevos objetivos antigénicos, como la proteína cinasa específica del músculo (MuSK) y la proteína 4 relacionada con el receptor de lipoproteínas de baja densidad (LPR4) (1).

La relación entre la miastenia grave y las enfermedades del timo es bien conocida. De 10 a $15 \%$ de los pacientes con la enfermedad tienen timoma, un tumor relativamente benigno, de crecimiento lento y de invasión local, mientras que en 60 a 70\% se evidencia hiperplasia folicular con proliferación de los centros germinales (1).

El timoma asociado a esta enfermedad se describe con frecuencia en pacientes aquejados de miastenia grave seropositiva (miastenia grave antiAChR) generalizada, con predominio de síntomas bulbares, la cual puede aparecer a cualquier edad, con un pico de inicio de los síntomas alrededor de los 50 años, y es igual de frecuente en hombres y mujeres $(3,4)$. En el cuadro 1 se describe la distribución del timoma en la miastenia grave según las clasificaciones de la gravedad clínica de Osserman y Genkins y de la Myasthenia Gravis Foundation of America (MGFA) $(5,6)$.

Además, en cerca de $95 \%$ de los timomas asociados a la miastenia grave se observan anticuerpos contra la titina y, en $70 \%$, anticuerpos contra el receptor de la rianodina; en pacientes menores de 40 años, la presencia de estos anticuerpos, o contra el fragmento recombinante de la titina de $30 \mathrm{kDa}$ (MGT30), indican la presencia de un timoma $(3,7)$.

Para correlacionar la histología y el curso clínico de los timomas, se han diseñado varias clasificaciones, entre las que la clasificación

\footnotetext{
$\overline{\text { Correspondencia: }}$

Lilliana María Giraldo, Calle 20 sur №35-35, Medellín, Colombia Teléfono: (574) 576 6666, extensión 91320

lilligiraldo@gmail.com

Recibido: 20/03/15; aceptado: 14/06/15
}

histológica de la Organización Mundial de la Salud y el sistema de estratificación clínico-patológico de Masaoka y Koga, son los que mejor reflejan el comportamiento oncológico del timoma, por lo cual, utilizados en conjunto, ofrecen una mayor precisión en el pronóstico (8). En cuanto a la clasificación histológica, la mayor incidencia de miastenia grave se describe en el timoma cortical total del subtipo B2, en el de predominio cortical o linfocítico del subtipo B1, y en el mixto del subtipo $A B$, en tanto que el timoma medular y el carcinoma tímico diferenciado o timoma epitelial o escamoso del subtipo A, tienen una incidencia menor de $10 \%(4,9)$.

El tratamiento de la miastenia grave se basa en cuatro opciones diferentes: los inhibidores de la colinesterasa, las inmunoterapias cortas como la plasmaféresis o la administración de inmunoglobulina intravenosa, la inmunoterapia prolongada con agentes inmunosupresores y la timectomía (7).

La timectomía es una modalidad de tratamiento que se aplica tanto para la miastenia grave como para los tumores del timo (10). En los pacientes con timoma la resección quirúrgica completa mediante una esternotomía mediana es el tratamiento de elección, independientemente de su estadio clínico. El uso de terapia adyuvante se recomienda para los estadios II a IV de la clasificación de Masaoka y Koga $(4,11)$, y en pacientes con miastenia grave, especialmente aquellos con la forma generalizada y moderada a grave de la enfermedad que no hayan mejorado con el tratamiento de primera línea y sean menores de 55 años (12). En el cuadro 2 se describe el sistema de clasificación del timoma propuesto por Masaoka y Koga.

\section{Caso clínico}

Se trata de una paciente de 43 años de edad a quien en el 2005 se le diagnosticó miastenia grave de clase $\mathrm{V}$ (según la clasificación de la MGAF), mediante examen clínico y prueba de fibra única al presentar una insuficiencia respiratoria después de la cirugía. Se halló una masa en el mediastino anterior que sugería la presencia de un timoma benigno, pero que, después de la timectomía transesternal, se confirmó por histología como un timoma cortical invasor de tipo B2 según la clasificación de la Organización Mundial de la Salud, y de estadio II, según la clasificación de Masaoka, por lo que recibió 38 sesiones de radioterapia adyuvante de 54 Gy. 
Cuadro 1. Distribución en los órganos del timoma según la gravedad clínica de la miastenia grave $(5,6)$

\begin{tabular}{|c|c|c|c|c|c|c|c|}
\hline \multicolumn{4}{|c|}{ Osserman- Genkins } & \multicolumn{4}{|c|}{ Myasthenia Gravis Foundation of America } \\
\hline \multicolumn{2}{|c|}{ Clase } & \multirow{2}{*}{$\begin{array}{l}\text { Definición } \\
\text { región ocular }\end{array}$} & \multirow{2}{*}{$\begin{array}{c}\begin{array}{c}\% \text { de } \\
\text { timoma }\end{array} \\
3\end{array}$} & \multicolumn{2}{|c|}{ Clase } & \multirow{2}{*}{$\begin{array}{l}\text { Definición } \\
\text { gión ocular }\end{array}$} & \multirow{2}{*}{$\begin{array}{r}\begin{array}{c}\% \text { de } \\
\text { timoma }\end{array} \\
1,93\end{array}$} \\
\hline I & & & & I & & & \\
\hline \multirow[t]{3}{*}{ II } & A & $\begin{array}{l}\text { Generalizada leve: } \\
\text { sin compromiso respiratorio }\end{array}$ & 34,5 & II & & $\begin{array}{l}\text { Músculos diferentes a los oculares, } \\
\text { compromiso ocular de cualquier gravedad }\end{array}$ & 27,1 \\
\hline & \multirow[t]{2}{*}{$\mathrm{B}$} & \multirow[t]{2}{*}{$\begin{array}{l}\text { Generalizada moderada grave: } \\
\text { con mayor compromiso bulbar }\end{array}$} & \multirow{2}{*}{53} & & A & $\begin{array}{l}\text { Leve: compromiso de predominio } \\
\text { apendicular, axial o de ambos }\end{array}$ & \\
\hline & & & & & B & $\begin{array}{l}\text { Leve: compromiso de predominio bulbar, } \\
\text { respiratorio o ambos }\end{array}$ & \\
\hline \multirow{2}{*}{\multicolumn{2}{|c|}{ III }} & \multirow{2}{*}{$\begin{array}{l}\text { Aguda respiratoria: compromiso } \\
\text { bulbar, axial y de extremidades, } \\
\text { con debilidad respiratoria }\end{array}$} & \multirow[t]{2}{*}{9,2} & \multirow[t]{2}{*}{ III } & A & $\begin{array}{l}\text { Moderada: compromiso de predominio } \\
\text { apendicular, axial o ambos }\end{array}$ & \multirow[t]{2}{*}{37,7} \\
\hline & & & & & B & $\begin{array}{l}\text { Moderada: compromiso de predominio } \\
\text { bulbar, respiratorio o de ambos }\end{array}$ & \\
\hline \multirow{2}{*}{\multicolumn{2}{|c|}{ IV }} & \multirow[t]{2}{*}{$\begin{array}{l}\text { Grave tardía: } \\
\text { compromiso general progresivo }\end{array}$} & \multirow[t]{2}{*}{-} & \multirow[t]{2}{*}{ IV } & A & $\begin{array}{l}\text { Grave: compromiso de predominio } \\
\text { apendicular, axial o de ambos }\end{array}$ & \multirow[t]{2}{*}{10,6} \\
\hline & & & & & B & $\begin{array}{l}\text { Grave: compromiso de predominio bulbar, } \\
\text { respiratorio o de ambos }\end{array}$ & \\
\hline $\mathrm{V}$ & & Asistencia respiratoria mecánica & - & V & & $\begin{array}{l}\text { Intubación, con asistencia respiratoria } \\
\text { mecánica o sin ella }\end{array}$ & - \\
\hline
\end{tabular}

Cuadro 2. Sistema de clasificación del timoma de Masaoka y Koga (11)

\begin{tabular}{cl}
\hline Estadio & Definición \\
\hline I & $\begin{array}{l}\text { Timoma encapsulado } \\
\text { II }\end{array}$ \\
III & $\begin{array}{l}\text { invasión de la grasa o pleura mediastinal, o } \\
\text { Invasión del mediastino }\end{array}$ \\
IV & $\begin{array}{l}\text { Diseminación en pleura o pericardio, o } \\
\text { diseminación linfática o hematógena }\end{array}$ \\
\hline
\end{tabular}

Durante los siguientes tres años, la paciente fue hospitalizada en varias ocasiones, en dos de ellas con crisis miasténicas que requirieron asistencia respiratoria mecánica y manejo con inmunoglobulina intravenosa por escasa mejoría con la plasmaféresis. La miastenia grave se trató con inhibidores de la colinesterasa en dosis diarias de 460 a $480 \mathrm{mg}$ de prednisolona en dosis de 40 a $60 \mathrm{mg} / \mathrm{día}$, e inmunosupresores: ciclofosfamida, $500 \mathrm{mg} / \mathrm{m}^{2}$ en dosis mensuales entre el $2005 \mathrm{y}$ el 2006, la cual se suspendió por vasculitis causada por hipersensibilidad; micofenolato de mofetilo en el 2007, el cual produjo cefalea intensa por lo que en el 2008 se cambió por azatioprina en dosis de 200 a 250 mg/día a la que la paciente mostró mayor tolerancia. En los controles del 2005, el 2008 y el 2009, con tomografías de tórax con contraste se evidenció una zona de engrosamiento de apariencia fibrosa que no captaba el medio de contraste, y no se registraron recurrencias.
Del 2009 a principios del 2013, la gravedad de los síntomas disminuyó (miastenia grave de clase IIllb, según la clasificación de la MGFA), y se continuó el tratamiento con $480 \mathrm{mg} /$ día de inhibidores de la colinesterasa y azatioprina en dosis de 200 a 250 $\mathrm{mg} /$ día. En del 2011, el control con la tomografía de tórax con contraste se evidenció nuevamente la zona de engrosamiento de apariencia fibrosa en el mediastino anterior sin cambios con respecto a los estudios previos.

Durante el 2013, la paciente presentó exacerbación de los síntomas (miastenia grave de clase IVb, según la MGFA), por lo que fue hospitalizada. La resonancia magnética de tórax con contraste reveló una lesión ovalada en el mediastino anterior, hiperintensa en T2 y por acción del contraste, y similar a la observada en el estudio del 2011, aunque, debido a las características magnéticas, esta vez indicaba un probable compromiso por timo residual o tejido cicatricial. El tratamiento neurológico se inició con inmunoglobulina intravenosa en una dosis de $2 \mathrm{~g} / \mathrm{kg}$ cada cuatro semanas, en tanto que los profesionales del grupo de radiología oncológica y de cirugía de tórax solicitaron una tomografía computadorizada en combinación con una por emisión de positrones (PET/CT), para descartar una lesión residual, de metástasis o de recurrencia, cuyos resultados no fueron concluyentes, por lo que se procedió a hacer una nueva resección quirúrgica sin que se encontrara evidencia histológica de recurrencia tumoral. 


\section{Discusión}

Hasta el $40 \%$ de los pacientes con timoma desarrollan una o más condiciones autoinmunitarias paraneoplásicas, de las cuales la miastenia grave es la más común (20 a $25 \%$ ); los anticuerpos contra los AChR son la principal causa de los síntomas en la miastenia grave asociada a timoma (3). Los síntomas iniciales comprometen sobre todo los músculos cervicales y tienen una distribución bulbar; cerca de $25 \%$ de los pacientes presentan una o más crisis respiratorias durante el curso de la enfermedad, principalmente durante los dos primeros años, siendo la insuficiencia respiratoria el signo de presentación en $1 \%$ de los casos $(2,13)$.

El tratamiento de elección en la miastenia grave asociada a timoma son los inhibidores de la acetilcolinesterasa, y más del $90 \%$ de los pacientes requieren de tratamiento inmunosupresor durante más de un año. En los casos graves con poca mejoría con el tratamiento farmacológico estándar y en presencia de una crisis miasténica, están indicadas la plasmaféresis y la inmunoglobulina, teniendo en cuenta que se debe intensificar en forma paralela el tratamiento farmacológico (3).

Entre las diferentes técnicas de imágenes diagnósticas que pueden ayudar a la detección y caracterización de los trastornos del timo, la tomografía computadorizada y la resonancia magnética son útiles para el diagnóstico del timoma, pero presentan limitaciones para diferenciar entre los diferentes subtipos histológicos establecidos por la Organización Mundial de la Salud y para establecer su pronóstico $(14,15)$. La combinación de la tomografía computadorizada y la tomografía por emisión de positrones (PET/CT), es superior a cualquiera de estas por separado, ya que la combinación de la información anatómica proporcionada por la primera y la información metabólica proporcionada por la segunda en un único entorno, permite mejorar la precisión diagnóstica al evidenciar metástasis o recurrencias (16).

La timectomía como modalidad de tratamiento se ha establecido tanto para los pacientes con miastenia grave como para aquellos con tumores del timo (15). Se ha estimado que, tras un periodo de seguimiento de 7,6 años, la timectomía es beneficiosa en el $86 \%$ de los pacientes con miastenia grave sin timoma, y que la edad, los hallazgos histológicos en el timo y la presencia de tejido ectópico son factores pronósticos significativos (12). En los casos de timomas en estadios II a IV la terapia adyuvante tiene un papel importante: la tasa de ausencia de recurrencia a los 15 años es del $100 \%$ para los tipos $A, A B$ y B1, y de 66,7 y $54,5 \%$, para los tipos B2 y B3, respectivamente, y de $66,7 \%$, para el tipo $\mathrm{C}$ a los 10 años (16).

Después de la timectomía, los pacientes con miastenia grave asociada a timoma pueden presentar una exacerbación de los síntomas, con un pico máximo a los 300 días de la resección (4). En la mayoría, la mejoría se inicia uno o dos años después del procedimiento, pero puede tardar hasta cinco o diez años. Además, la reaparición de síntomas de miastenia grave no necesariamente implica una recurrencia del tumor (12).

En el caso de esta paciente con miastenia grave asociada a timoma, no se presentaron cambios sustanciales en las manifestaciones clínicas, ni se pudieron reducir los medicamentos para tratarla tras nueve años de la resección tumoral, debido a la aparición de síntomas que no correspondían a la presencia de tejido residual o recurrencia de la enfermedad.

\section{Conflicto de intereses}

Los autores declaran que no tienen ningún tipo de conflicto de intereses. La información utilizada fue proporcionada por el Instituto Neurológico de Colombia y contó con la aprobación del Comité de Ética de la institución.

\section{Financiación}

Al tratarse de un reporte de caso, no se incurrió en gastos monetarios, por lo que no se requirió ningún tipo de financiación.

\section{Referencias}

1. Berrih-Aknin S, Le Panse R. Myasthenia gravis: A comprehensive review of immune dysregulation and etiological mechanisms. J Autoimmun. 2014;52:90-100. http://dx.doi.org/10.1016/j.jaut.2013.12.011

2. Suh J, Goldstein JM, Nowak RJ. Clinical characteristics of refractory myasthenia gravis patients. Yale $\mathrm{J}$ Biol Med. 2013;86:255-60

3. Romi F. Thymoma in myasthenia gravis: From diagnosis to treatment. Autoimmune Dis. 2011;2011:474512. http:// dx.doi.org/10.4061/2011/474512

4. Zieliński M. Management of myasthenic patients with thymoma. Thorac Surg Clin. 2011;21:47-57. http://dx.doi. org/10.1016/j.thorsurg.2010.08.009

5. Osserman KE, Genkins G. Studies in myasthenia gravis: Review of a twenty-year experience in over 1200 patients. Mt Sinai J Med NY. 1971;38:497-537. 
6. Jaretzki A, Barohn RJ, Ernstoff RM, Kaminski HJ, Keesey JC, Penn AS, et al. Myasthenia gravis: Recommendations for clinical research standards. Task Force of the Medical Scientific Advisory Board of the Myasthenia Gravis Foundation of America. Neurology. 2000;55:16-23.

7. Sieb JP. Myasthenia gravis: An update for the clinician. Clin Exp Immunol. 2014;175:408-18. http://dx.doi.org/10.1111/ cei. 12217

8. Marx A, Willcox N, Leite MI, Chuang W-Y, Schalke B, Nix W, et al. Thymoma and paraneoplastic myasthenia gravis. Autoimmunity. 2010;43:413-27. http://dx.doi.org/10. 3109/08916930903555935

9. Rosai J, Sobin LH. Histological typing of tumours of the thymus. Berlin: Springer; 1999.

10. Fujii Y. Thymus, thymoma and myasthenia gravis. Surg Today. 2013;43:461-6. http://dx.doi.org/10.1007/s00595012-0318-2

11. Masaoka A, Monden Y, Nakahara K, Tanioka T. Follow-up study of thymomas with special reference to their clinical stages. Cancer.1981;48:2485-92.
12. Hammoumi ME, Arsalane A, Oueriachi FE, Kabiri EH. Surgery of myasthenia gravis associated or not with thymoma: A retrospective study of 43 cases. Heart Lung Circ. 2013;22:738-41. http://dx.doi.org/10.1016/j. hlc.2013.02.010

13. Blichfeldt-Lauridsen L, Hansen BD. Anesthesia and myasthenia gravis. Acta Anaesthesiol Scand. 2012;56: 17-22.

14. Otsuka $\mathbf{H}$. The utility of FDG-PET in the diagnosis of thymic epithelial tumors. J Med Investig. 2012;59:225-34.

15. El-Bawab H, Al-Sugair AA, Rafay M, Hajjar W, Mahdy M, Al-Kattan K. Role of flourine-18 fluorodeoxyglucose positron emission tomography in thymic pathology. Eur $\mathrm{J}$ Cardiothorac Surg. 2007;31:731-6. http://dx.doi.org/10.1016/j. ejcts.2007.01.024

16. Rashid OM, Cassano AD, Takabe K. Thymic neoplasm: A rare disease with a complex clinical presentation. J Thorac Dis. 2013;5:173-83. http://dx.doi.org/10.3978/j.issn.20721439.2013.01.12. 